\title{
ESTUDIOS
}

\section{Jicoténcal, Primera Novela Histórica en Castellano}

Dos años antes de que apareciera en París la novela Ramiro, Conde de Lucema, de Rafael Húmara, ${ }^{1}$ se había publicado en Filadelfia, Estados Unidos, una obra titulada Jicotencal, ${ }^{2}$ cuyo asunto, la conquista de México por Hernán Cortés, es histórico. A dicha obra - ya que está escrita en castellano y lleva la fecha de I826-y no a la de Húmara (publicada en 1828) corresponde el título - mientras no se descubra otra de fecha anterior- de primera novela histórica en castellano. Desgraciadamente, el autor no nos dejó su nombre. La importancia que la obra tiene en la historia del género nos obliga, sin embargo, a reanudar nuestros esfuerzos para determinar el nombre de su autor. Aunque ya se ha escrito bastante sobre el tema, hasta hoy no se ha llegado a una conclusión definitiva. Los críticos que del asunto se han ocupado se concretan a discutir si el autor es español o hispanoamericano, sin proponer el nombre de ningún escritor como posible autor de la novela. Es nuestro intento en este estudio de presentar los nombres de varios autores, lo mismo que investigar la posibilidad de que uno de ellos sea el autor de Jicoténcal. Mas antes de entrar en materia nos parece conveniente hacer una descripción de la obra, hasta hoy casi desconocida debido a la escasez de los ejemplares.

Al examinar la novela descubrimos que, según reza el pie de imprenta, la obra, en dos tomos de tamaño pequeño, $13.5 \times 8.5 \mathrm{cms}$, y de. $224 \mathrm{y}$

1 Sobre la novela histórica en España ver Guillermo Zellers, La novela bistórica en España. 1828-1850, Nueva York, 1938.

${ }_{2}$ En el original, Jicontencal, sin acento, no porque se pronunciara como voz aguda sino debido al sistema de acentuación característico de la época. 
247 páginas respectivamente, fue publicada en Filadelfia en la imprenta de Guillermo Stavely el año de I826. Podríamos empezar dudando que la obra fuera publicada en Filadelfia. Dicha duda nos la sugiere el saber que otra obra, que también lleva como lugar de publicación la ciudad de Filadelfia (escrito Philadelphia), fue realmente publicada en La Habana. Nos referimos a la obra Bosquejo ligerísimo de la revolución de Megico, deside el grito de Iguala basia la proclamación imperial de Iturbide, por Un Verdadero Americano. . Philadelphia, Imprenta de Teracrouef y Naroajeb, I822. Es evidente aquí que los nombres de los impresores son ficticios. $Y$ en verdad, Teracrouef y Naroajeb es el seudónimo de Rocafuerte y Bejarano, verdadero autor de este curioso libro publicado en La Habana y no en Filadelfia. ${ }^{3}$ No ocurre lo mismo, sin embargo, con ficoténcal. En Filadelfia existió un impresor y editor llamado William Stavely, quien publicaba el Episcopal Recorder en 12 Pear y que vivía, años más tarde, en el número 474 de la calle S. Front. ${ }^{4}$ Este editor publicó, además de la obra que nos ocupa, otras en castellano, de las cuales ya daremos razón.

Es de interés notar que Jicoténcal fue registrado, para los derechos literarios, por un tal Frederick Huttner, según se lee en la página 4: "Eastern Distric of Pennsylvania, to with: Be it remembered, that on the eighteenth day of August, in the fifty-first year of the Independence of the United States of America, A. D. 1826. Frederick Huttner, of the said District, hath deposited in this office the title of a book, right whereof he claims as Propietor, in the words following, to wit: JICOTENCAL..." De lo cual se desprende que el libro pertenecía a Huttner, ya fuera por haberlo escrito 5 o por haber comprado el manuscrito. En Filadelfia vivieron dos Frederick Huttner, el uno (Hutner, con una sola $t$ ) profesor de música, con residencia en 264 High y el otro, médico de profesión en 239 Spruce. $^{6}$

La novela, según se anuncia en la primera página, estaba de venta en

3 Bachiller y Morales hizo el siguiente comentario sobre este opúsculo: "Severísimo escrito contra Iturbide que termina con un poema de Heredia, que no lo firma". También nos dice que "aunque parece impreso en Filadelfia lo fué en La Habana". (Joseph Sabin, Biblioteca Americana, Nueva York, 1868-1936, vol. II, 1869, p. 299, No. 6456 ).

4 Desilver's Pbiladelpbia Directory and Stranger's Guide, 1835 and 1836. Philadelphia, 1835.

5 Hay quien se lo adjudique. Ver. G. A. Gómez, Xicotencatl Axayacatzin (Bosquejo biográfico de un gran patriota), México, D. F., 1945, "Bibliografia".

6 Ver A. M'Elroy's Philadelphia Directory for the Year 1837. 
Filadelfia "en casa del Señor F. Merino, profesor de lengua castellana, en el Instituto de Franklin; y en la del Señor J. Laval, No. Ir8, Chestnut Street. $Y$ en Nueva York, en casa de Lanuza y Mendía, No. 3. Varick street". Vivía en Filadelfia, por aquellos años un Félix Merino, "teacher and translator of Spanish and French languages" con residencia en el número 88 de la calle Wood. ${ }^{7}$ El J. Laval era John Laval, impresor también residente en Filadelfia. Lanuza y Mendía eran libreros en Nueva York: En la última página del tomo segundo de Jicoténcal encontramos el siguiente interesante anuncio: "Libros de fondo que se encuentran en casa de Lanuza y Mendía de Nueva York: Diccionario filosófico de Voltaire, traducido por C. Lanuza, Io tom. en $18^{\circ} \mathrm{N}$. York, 1825; Cuentos y Sátiras de Voltaire, puestos en verso castellano por $\mathrm{M}$. Domínguez, un tomo en $18^{\circ} \mathrm{N}$. York 1825; El Vicario de Wakefield, por el Dr. Goldsmith, traducido por M. Domínguez, un tomo en $18^{\circ} \mathrm{N}$. York, 1825; Vida de Jorge Washington, por Ramsey, 2 tom. en $18^{\circ} \mathrm{N}$. York r825; Compendio de la bistoria de los Estados Unidos, un tomo en $18^{\circ} \mathrm{N}$. York $\mathrm{I} 825$; Auxiliar Vocabulario de bolsillo español inglés, por J. L. Barry, un tom. en $16^{\circ} \mathrm{N}$. York, I825; Fábulas de Samaniego, un tom. en $18^{\circ} \mathrm{N}$. York I826; Ortografia de la lengua castellana, por la Academia española, un tom. en $18^{\circ} \mathrm{N}$. York I826; Jicoténcal, 2 tom. en $\mathrm{I} 8^{\circ}$ Filadelfia $\mathrm{I} 826$. EN PRENSA, Vida de Benjamin Franklin, escrita por él mismo; Clotilde, o el Médico Confesor, por Víctor Ducange; Persihes y Sigismunda, última obra de Cervantes.-También se hallará un surtiło de libros españoles antiguos y modernos y toda clase de encuadernación". En 1828, Lanuza Mendía y Cía. publicaron en Nueva York las Poesias de un mexicano (2 vols. en $8^{\circ}$ ) de don Anastasio Matía de Ochoa, autor también de "una novela de costumbres mexicanas, de la cual ni el nombre ha quedado". ${ }^{8}$

Por lo anterior nos damos cuenta de que Jicoténcal estaba de venta en Nueva York el mismo año que vió la luz en Filadelfia. En febrero del año siguiente el poeta William Cullen Bryant le dedica una larga reseña en su revista The United States Review and Literary Gazette, ${ }^{9}$ que por aquel entonces publicaba en Boston. Tenemos noticias de que el libro lle. gó a México en fecha temprana, aunque no creemos que haya tenido amplia circulación. En la lista de libros que pertenecieron al poeta cubano

7 Desilver's Directory, 1836.

8 Ver Francisco Pimentel, "Novelistas y oradores mexicanos", Obras completas, México, 1904, V. 296.

9 Vol. I, No. 5, feb., 1827, pp. 336-346. Ejemplar en la biblioteca de Enory University. 
José María Heredia encontramos el título Xicotencal, clasificado entre las novelas. ${ }^{10}$ El mismo poeta no habla de ella en sus escritos. En 1828 aparecen en Puebla la tragedia en cinco actos Tewila de Ignacio Torres Arroyo $^{11}$ y el drama Xicobténcatl de José María Moreno Buenvecino. ${ }^{12} \mathrm{Al}$ año siguiente, también en Puebla, José María Mangino publica su comedia en cinco actos Xicoténcatl. ${ }^{13}$ Las tres obras son el resultado de un concurso celebrado en Puebla en 1828, concurso inspirado por la novela de Filadelfia. ${ }^{14}$ En las obras publicadas de 1829 en adelante, y en las cuales aparece Teutila, ya no nos es posible decir si la influencia procede directamente de la novela de Filadelfia o de las obras dramáticas publicadas en Puebla ${ }^{15}$ Teutila -el nombre se deriva de Teutile, general mexicanoes un personaje creado por el autor de Jicoténcal. ${ }^{\mathbf{1 6}}$

Pasemos a tratar del contenido de la novela. El tema es la conquista de México por Hernán Cortés y los tlaxcaltecas, sus aliados. Los personajes históricos, además de Cortés, son Xicoténcatl el joven-héroe de la novela-, Xicoténcatl el viejo, Maxiscatzin, doña Marina, Diego de Ordaz, Moctezuma y Fray Bartolomé de Olmedo. En esta parte el autor anónimo sigue fielmente el relato de Solís a quien cita con frecuencia. En nota a la página 14 del primer tomo, nos dice: "Todo lo que en el discurso de esta obra irá con letra cursiva, será copiado literalmente de la Historia de la conquista de Méjico, por don Antonio de Solís, que es el escritor mảs entusiasta de las prendas y méritos de Hernán Cortés". Comparando dichas

10 Ver "La biblioteca de José Ma. Heredia", Romance, México, I, 7, mayo 1 de 1940, p. 22. El manuscrito del Catálogo de los libros de Heredia se conserva en la Biblioteca Nacional de Cuba. Ver José Antonio Fernández de Castro, "Latra y algunos románticos de América", Revista de la Biblioteca Nacional, La Habana, I, No. 3, mayo, 1950 , p. 189.

11 Ignacio Torres Arroyo, Teutila; tragedia en cinco actos. Puebla, Oficina del C. Pedro de la Rosa, 1828. 83 pp. Dedicada al Ciudadano General de División Vicente Guerrero. (Ejemplar en el British Museum, No. 11726. c). En el Catalogue de la ricbe bibliotbeque de José Maria Andrade, Leipsig-París, 1869, aparece un ejemplar de esta obra bajo el nombre de Arroyo, J. T. (núm. 3855). Allí también se registra un ejemplar de nuestro Jicotencal (núm. 3974).

12 José Maria Moreno Buenvecino, Xicobtencatl; tragedia en cinco actos. Puebla, Imp. del Patriota, $1828.56 \mathrm{pp}$. (Ejemplar en la Biblioteca Nacional de México).

13 José María Mangino, Xicotencall; comedia heroica en cuatro actos, compuesta y ordenada con un coro de música. Puebla, Pedro de la Rosa, 1829. 90 pp. (Ejemplares en la Universidad de Yale y en la Biblioteca Pública de Nueva York). 14 Ver Daniel Wogan, "The Indian in Mexican Dramatic Poetry (18231918)", Bulletin of Hispanic Studies, XXVII (1950) 164; también D. W. McPheeters, "Xicoténcatl, símbolo republicano y romántico", NRFH, X (1956), 408-409.

15 Para un estudio de estas obras véase el trabajo de McPheeters.

116 A pesar de ello, hay quien lo considere personaje histórico. Ver G. A. Gómez, op. cit., pp. $21,24,28,31,32,45,47$, etc. 
citas con el original de Solís, hallamos que el novelista sigue fielmente al historiador. Solamente de él se desvía al tratar de la matanza de los nobles mexicanos en el Templo Mayor. En la nota que pone en la página 105 del tomo segundo, en donde se relata el episodio, advierte: "En la relación de este hecho se sigue al venerable frai Bartolomé de las Casas". Esta parte histórica termina con la muerte de Xicoténcatl, a quien Cortés manda ahorcar en Texcoco. El Conquistador se prepara para atacar y tomar la Gran Tenochtitlán.

A la par del tema histórico se desarrolla el amoroso, esto es, el idilio entre Teutila y Xicoténcatl el joven. Al mismo tiempo, el joven Diego de Ordaz, generoso y bien intencionado, ama secretamente a Teutila y es amado, no tan secretamente, por doña Marina. La novela termina con el auto-envenenamiento de Teutila - muere dramáticamente frente a Cortés, a quien pensaba asesinar-y el arrepentimiento de doña Marina.

El fin primordial del autor, sin embargo, parece ser la prédica política. Así lo comprendió su primer crítico, el poeta Bryant, al decir: "It has the merit of containing pretty just and enlightened notions on political government and other important subjects, and to inculcate these, we suppose, was the principal aim of the writer" (pp. 336-337). La narración, sin embargo, no le parecía muy bien urdida: "As a story we cannot give it the praise of being very skilfully contrived, although it is written with about the same degree of talent as the mass of modern works of the kind" (p. 336).

Aunque parezca increíble, la reseña de Bryant es la única que conocemos escrita durante el siglo xIx. Tal vez existan otras. Sin embargo, hasta hoy nuestras pesquisas han sido infructuosas. En verdad, fuera de la reseña de Bryant y la cita que de ella hace el dramaturgo Mangino-de la cual ya nos ocuparemos - la novela no vuelve a ser mencionada por la crítica sino hasta nuestros días. La obra fue descubierta, podría decirse, por el profesor Read al escribir su tesis doctoral en la Universidad de Columbia, cuya biblioteca posee un ejemplar de ficoténcal. ${ }^{17}$ Desde esa fecha en adelante, ya no se puede hablar del desarrollo de la novela his. panoamericana sin mencionarla. A pesar de su importancia, todavía no se ha descubierto el nombre del autor. He aquí lo que la crítica opina sobre la paternidad literaria de la obra.

17 John Lloyd Read, The Mexican Historical Novel (1826-1910). Nueva York, Instituto de las Españas, 1939. 
El poeta Bryant, su primer crítico, nos dejó una idea bastante ambigua sobre la posible nacionalidad del autor. Hela aquí: "Jicotencal, his wife his father, and the Mexican general Teutile, are very unprejudiced, enlightened, and philosophical savages, and in their notions of government and religion, approach very nearly to the modern liberales of Spain... The character of Cortés is more fairly although very unfavorably represented... The author of jicotencal seems to have made ample allowance for the partiality of his countrymen in their views of the character of Cortés, and has busied hunself, without remorse, in bringing out and placing in a strong light its darker features" (p. 343). Lo que puede indicar que el autor es español o mexicano, según se interprete la palabra "countrymen" ya que tanto los españoles como los mexicanos son parciales en la inter. pretación del carácter de Cortés. Al profesor McPheeters le parece que la cita indica que el autor era español. A nosotros, sin embargo, nos parece que Bryant creía que el autor era mexicano. Nos hace pensar en ello la primera frase de la reseña: "We have been principally induced to notice this book on account of its belonging to the Spanish literature of America" (p. 336).

Los críticos que han creído que el autor es mexicano son Pedro Henríquez Ureña, Ralph Warner y José Rojas Garcidueñas. ${ }^{18}$ Henríquez Ureña basó su juicio en lo exaltado del patriotismo indígena que encontramos en la obra: "Y la especie de patriotismo indígena que alienta en la obra hace pensar que el autor ha de ser mexicano". Algunos años más tarde, repite la idea: "El autor anónimo fue, probablemente, un mexicano... Y hay una especie de patriotismo indio en la novela". 19 Warner, sin embargo, no está muy seguro de que el autor sea mexicano: "No estoy seguro -nos dice- que sea el autor mexicano, pero no sería imposible dado el número de mexicanos en los Estados Unidos y en Filadelfia en aquellos tiempos". Rojas Garcidueñas sigue en su opinión a Henríquez Ureña. Sin afirmar que el autor sea mexicano, defiende la tesis de su procedencia hispanoamericana. La tesis de Henríquez Ureña, en nuestro concepto, no puede ser defendida. He aquí el porqué.

En 1829, el dramaturgo Mangino observó los pocos conocimientos

18 Véase la reseña del libro de Read por Henríquez Ureña en la Revista de Filología Hispánica, Buenos Aires, IV (1942), 188-189; también Ralph Warner, Historia de la novela mexicana en el siglo XIX, México, 1953, p. 10; José Rojas Garcidueñas, "Jicotencal, una novela histórica hispanoamericana precedente al romanticismo español", Anales del Inst. de Inv. Estéticas, México, 24, 1956, pp. 53-76.

19 Pedro Henríquez Ureña, Las corrientes literarias en la América Hispánica, $2^{\sharp}$ ed., México, 1954, p. 128; también la nota 24, p. 243. (1a. ed. en inglés, 1945). 
que el autor demuestra, en la novela, de la topografía mexicana; en la página 89 de su obra se halla este comentario: "Los mamarrachos y deformidades de que abunda dicha obra son insufribles, y mucho más los ningunos conocimientos del país, pues el autor nos describe a un país arenoso, seco y árido como es Acatzingo, lleno de frondosas arboledas, hermosísimos arroyos, amenísimos prados; y sobre todo... (nos dice) que Xicoténcatl iba todas las noches desde Tlaxcala a Acatzingo... Si el autor supiera que desde Tlaxcala a Acatzingo hay, lo menos dieciocho leguas, y una elevadísima montaña que atravesar, vería que era imposible que un hombre anduviese treinta y seis leguas diarias, y a pie, ocupando en esta tarea la noche, y el día en los deberes de su alto destino..."20 A lo anterior puede agregarse una razón más convincente: el poco o ningún conocimiento que el autor tenía de las palabras indígenas de uso corriente en México. Las pocas que hemos encontrado - yopales, calpisca, copal, tamemes- están usadas en párrafos sacados de Solís. Lo que denota que el autor no es mexicano es el uso de la palabra yopales. Solís, en el capítulo XVI del libro II de su obra dice: "Estaban los senadores sentados por su antigüedad sobre unos taburetes bajos de maderas extraordinarias, hechos de una pieza, que llaman yopales". Y el autor de Jicoténcal: "Estaban sentados los individuos por su antigüedad en unos taburetes bajos, de maderas exquisitas y de una sola pieza, que se llama yopales" (I, I3). Dudamos mucho que un mexicano no sepa que estas sillas se llaman equipales y no yopales. A lo que hay que añadir que en Jicoténcal se usa la $j$ para escribir los nombres mexicanos. Don Carlos María de Bustamante, en 18302 hacía la siguiente observación sobre el uso de esta letra: "Jamás, jamás los nombres mexicanos se escriben con $j$ siempre con $x \circ(g)$; el extravagante de Mora proscribió la $x$ en la obra del padre Clavijero, y no puede leerse sin vomitar su traducción"21 En la novela de Filadelfia se escribe Jicoténcatl, Méjico, mejicano, etc. La única excepción es el nombre del senador tlaxcalteca Magiscatzin. Hay que hacer notar que el autor usa la grafía $g$ frente a $e$ en palabras españolas como extrangero, gefe, magestad, bagage, etc. Además, en los nombres toponímicos Zocothlan y Olintetb el uso de la $t h$, como ya observó Rojas Garcidueñas (p. 70 ) no tiene sonido especial en náhuatl y su uso parece ser arbitrario e indica desconocimiento de esa lengua. Otros nombres propios de origen mexicano están castellanizados de acuerdo con la pronunciación de la época de la conquista, debido a que

20 Citado por McPheeters, p. 408.

21 En nota a la Historia de Sahagún, ed. de Bustamante, México, 1830, vol. III, p. 248. 
están sacados de Solís, quien los copió de cronistas del siglo xvi. Entre otros: Tlascala, Popocatepec, Guatimotzin, Quetlabaca, Cualpopoca, etc. En cuanto a otemies ( $\mathrm{I}, 9)$, en vez de otomies, tal vez sea debido a error tipográfico, no escasos en el libro.

Mas si el autor no es mexicano, forzoso nos es decir que tampoco es español. La mayor parte de los críticos que del asunto se han ocupado parece inclinarse a la teoría del origen hispanoamericano del autor. Solamente Anderson Imbert y Lloréns Castillo han discutido la posibilidad de que sea español. El primero basa sus afirmaciones en las siguientes razones:

Por encima de las fronteras politicas, los liberales de España y de Hispanoamérica trabajaron juntos. La lucha contra el absolutismo los unia. Aun los temas de la Independencia americana, de las tradiciones indigenas y de la condenación a los conquistadores eran comunes. No niego la posibilidad de que un mexicano escribiera Jicoténcatl; lo que digo es que sus censuras a Hernán Cortés están inspiradas no por patriotismo mexicano, mucho menos por espíritu indigenista, sino por las ideas racionalistas, humanitarias y liberales de la Ilustración. El autor, quien quiera que fuera, eligió Tlascala como escenario, y a Jicoténcal como héroe, porque esa realidad se prestaba mejor que ninguna otra a su ideología afrancesada. Tlascala es la República; Cortés y Moctezuma, los déspotas; los dos Jicoténcal, el viejo y el joven, simbolizan la libertad, la virtud, la razón; Teutila, la inocencia. Ni siquiera hay color americano en ficoténcal; paisajes convencionales, pocas palabras indigenas, apenas trazos costumbristas en las nupcias de Jicoténcal y Teutila... Es novela discursiva, no descriptiva, y los discursos traducen los hechos de la conquista de México a términos europeos... A Cortés se lo infama, no por ser español, sino por ser tirano... El autor, pues, es más liberal que patriota, más racionalista que indianista, más universal que nacionalista. Las notas indianistas son también europeas: era el tema del indio inocente... Pero mientras no se resuelva este misterio del autor anónimo es legitimo incluir Jicoténcal en la historia de la literatura hispanoamericana. ${ }^{22}$

En fecha posterior, Anderson Imbert expone la misma hipótesis, aunque con menos seguridad en cuanto a la clasificación de la novela. Lo que en la cita anterior es una afirmación, se convierte aquí en una interrogante; ¿Es legítimo incluir Jicoténcal en una historia de la literatura hispanoamericana?'23

22 Enrique Anderson Imbert, "Notas sobre la novela histórica en el siglo XIx", La novela bispanoamericana, ed. a cargo de Arturo Torres Ríoseco, Albuquerque, N. M., 1952, pp. 5-6. (Memoria del V. Congreso del Inst. Intern. de Lit. Iberoamericana).

23 Enrique Anderson Imbert, Historia de la literatura bispanoamericana, México, $2^{2}$ ed., 1957 , p. 132. 
El único crítico que ha seguido la hipótesis de Anderson Imbert es Lloréns Castillo. Al hablar de los escritores que colaboraron en la revista El Aguimuldo, publicada en Filadelfia en 1829, nos dice: "Heredia da a entender que El Aguinaldo estaba redactado principalmente por emigrados españoles. La lectura del correspondiente a I830, único que conozco, parece confirmarlo... De este grupo de Filadelfia salió en 1826 Jicoténcal, la primera novela histórica en español, cuya atribución a autor americano, aunque parezca lo más probable, no es fácil deducir. A sospechar que fue español se inclina, con buenas razones, Enrique Anderson Imbert. .."24

Ya Rojas Garcidueñas (pp. 73-74) se ocupó de demostrar que había grandes diferencias en la ideología de los españoles y los hispanoamericanos de la época de las guerras de Independencia. Para apoyar sus asercio. nes, el autor cita el estudio de Lloréns Castillo, y en verdad este autor ha demostrado que existían dichas diferencias en el pensar de los librales españoles e hispanoamericanos. Al hablar de los emigrados españoles y sus relaciones con los americanos (pp. 248-25r) hace la siguiente observación:

El equívoco fundamental consistió en que los liberales españoles estuvieron dispuestos desde el principio a dar a los americanos todas las libertades menos la independencia, que era a lo que aspiraban éstos en primer término, con libertades o sin ellas.

También cita un juicio que apareció en El Español Constitucional en febrero de I825, en el cual los redactores "creen su deber rebatir con la misma sinceridad algunos de los cargos que se han venido haciendo 'por una rutinaria tradición' contra los españoles en general... Viejas son las acusaciones contra España por la conquista y colonización de América, pero las cosas han llegado a tal punto que 'apenas hay quien no se permita denigrar el ilustre nombre de Hernán Cortés'. No se trata de justificar sus usurpaciones ni sus violencias, sino de tener en cuenta la aspereza de los tiempos en que vivieron aquellos conquistadores".25 A pesar de que Lloréns Castillo nos dice, como ya vimos, que ficoténcal salió del grupo de

24 Vicente Lloréns Castillo, Liberales y románticos, una emigración española en Inglaterra (1823-1834), México, 1954, p. 212, nota 50. Sobre El Aguinaldo ver McPheeters, op. cit., p. 406, nota 14 .

25 Ibid., D. 249 . Véanse también las pp. 261-263, en las cuales se discuten las rencillas entre los españoles emigrados y los hispanoamericanos; en la p. 263 leemos: "Pero lo ocurrido vuelve a poner de manifiesto lo inestable y ambigua relación existente entre los liberales y los americanos. La independencia era muy reciente y la hostilidad nutua no había desaparecido. Algunos, como hemos visto, intentaron la reconciliación. Pero no faltaban entre los emigrados en Londres quienes seguían calificando de insurgentes a los americanos; mientras no pocos de éstos persistian en su hostilidad a todo español". 
escritores españoles emigrados que en Filadelfia publicaban El Aguinaldo, en la página anterior (2II) nos había dicho que "la novela no fue cultivada por los emigrados más que en lengua inglesa; pero las breves composiciones del No me olvides of recen ejemplos de la leyenda romántica en prosa y la estampa costumbrista. En estos años de tan extrema indigencia en la prosa narrativa española constituyen una excepción..."

A las anteriores vamos a agregar otras observaciones que nos han hecho pensar que el autor no fue español. Al hablar Solís de la matanza de los nobles mexicanos en el Templo Mayor, dice: "Los escritores forasteros se apartan más de lo verosímil, poniendo el origen y los motivos de aquella turbación entre las atrocidades con que procuran desacreditar a los españoles en la conquista de las Indias; y lo peor es que apoyan su malignidad citando al padre fray Bartolomé de las Casas o Casaus, que fue después obispo de Chiapa, cuyas palabras copian y traducen, dándonos con el argumento de autor nuestro y testigo calificado... Los más de nuestros escritores le convencen de mal informado en esta y otras enormidades que dejó escritas contra los españoles" (lib. IV, cap. xii). El autor de Jicotén$c a l$, al hablar de este episodio, hace precisamente lo que Solís dice que hacen los escritores forasteros, esto es, citar a Las Casas. En nota a la página I05 del segundo tomo leemos: "En la relación de este hecho se sigue al venerable frai Bartolomé de las Casas". Tal vez haya puesto la nota precisamente por lo que dice Solís, para demostrar que no era español.

Fáltanos agregar dos notas que tal vez sean significativas. En la página 65 del segundo tomo de la novela hallamos esta frase: "Esta loca de la casa, como la llama una santa española". Si el autor fuera español, ¿llamaría a Santa Teresa "un santa española"? Parece que aquí se ve lo español con perspectiva de extranjero. También hemos notado que al citar a Solís se cambia el pronombre le en lo; dice Solís: "Pero el padre fray Bartolomé de Olmedo le puso en razón" (Lib. III, cap. iv). Y el autor anónimo transcribe: "Y aun refiere el historiador más apasionado suyo, que el capellán lo puso en razón" (I, r59).

Aunque no es esencial, es muy probable que el autor de la novela que nos ocupa haya vivido en los Estados Unidos, sobre todo en Filadelfia, o Nueva York. He aquí una lista parcial de los hispanos que vivieron en esa región por los años en que se publicó Jicoténcal: los cubanos Domingo del Monte, 'Tomás Gener, Félix Varela, Leonardo Santos Suárez, Juan Gualberto Ortega, Luciano Ramos, Gaspar Betancourt Cisneros, José de la Luz 
Caballero, José María Heredia, José Antonio Saco, Juan de Costa, José Teurbe Tolón, Miguel María Caraballo, Agustín Hernández, José Luis Alfonso y García y Silvestre Luis Alonso y Soler;26 los mexicanos Félix Merino, Fray Servando Teresa de Mier, Lorenzo Zavala y Anastasio Zerecero; los colombianos José Fernández Madrid, Juan Garca del Río, Manuel Torres (aunque nacido en España) y José María Salazar; el venezolano Nicanor Bolet Peraza; el argentino José Antonio Miralla; el peruano Manuel Lorenzo Vidaurre; el ecuatoriano Vicente Rocafuerte y los españoles Pedro de Estala y Félix Mejía; además algunos cuya nacionalidad no hemos podido identificar: Manuel Domínguez, José Gertrudis Pinzón, Francisco de la O. García, Melitón Lamar, Cirilo Ponce, Mariano Torrero, C. Lanuza y Juan de Otero.

De la anterior lista eliminaremos a los que no son escritores. A los mexicanos y a los españoles, por las razones arriba expuestas, también los podemos eliminar. Nos quedamos, por lo tanto, con los siguientes: Del Monte, Varela, Lanuza, Ortega, Luz Caballero, Heredia, Saco, Teurbe Tolón, Fernández Madrid, Bolet Peraza, Miralla, Vidaurre, Rocafuerte, Domínguez y Betancourt Cisneros.

Luz Caballero no vivió en los Estados Unidos antes de I828; su primer viaje lo hizo en mayo de ese año. ${ }^{2 \tau}$ Lo mismo acontece con Del Monte, quien en 1827 hizo un viaje a España y de paso se detuvo en Nueva York para visitar a su maestro el P. Varela. Dos años más tarde, en Filadelfia, publicó los Versos de J. Nicasio Gallego, en la imprenta del "Mensajero". 28 Es interesante notar que en el erudito estudio de Del Monte sobre la novela histórica (Escritos, II, 2II-244), en el cual discute las primeras obras en el género publicadas en París y España, lo mismo que otras insignificantes manifestaciones del mismo, no menciona la novela de Filadelfia. ¿Acaso ignoraba su existencia? No creemos, sin embargo, que sea obra suya, ya que su estilo es enteramente distinto del de ficoténcal. En la ideología, y sobre todo en las ideas políticas, hay grandes divergencias.

Dominguez, Lanuza y Ortega nos parece que fueron simples traductores. El primero es el traductor de los Cuentos y sátiras de Voltaire y del

26 Ver Ernest E. Moore, "José María de Heredia in the United States and Mexico", Modern Language Notes, LXV (1950), 41-46; Ernest R. Moore, "José María Heredia in New York, 1824-1825", Symposium, V (1951), 256-291. p. 385 .

27 Ver Francisco Calcagno, Diccionario biográfico cubano, New York, 1878,

28 Ver José A. Fernández de Castro, Escritos de Domingo del Monte, 2 tomos, La Habana, 1929. 
Vicario de Wakefield, ambas obras publicadas en Nueva York en $1825^{20}$ Lanuza, librero de Nueva York, publicó en esa ciudad en I825 el monumental Diccionario de Voltaire. ${ }^{30}$ Ortega "tradujo del alemán la tragedia en cinco actos, de Kotzebue, titulada: Pizarro o los peruanos, Habana, r822" (Calcagno).

Aunque el venezolano Nicanor Bolet Peraza y el argentino José Antonio Miralla vivieron en los Estados Unidos, no sabemos que hayan residido en Filadelfia o publicado libros en esa ciudad. El primero fundó y dirigió dos revistas en Nueva York, La América y la Revista Ilustrada, en las cuales publicaba interesantes cuadros de costumbres, lo mismo que relatos y cuentos breves de escritores norteamericanos. ${ }^{31}$ El inquieto Miralla, poeta y traductor de las Cartas de Jacopo Ortis (Habana, 1822) de Foscolo y de la elegía En el cementerio de una aldea de Thomas Gray, compañero de Heredia en Nueva York y su preceptor en el aprendizaje de la lengua inglesa, murió en México un año antes de que apareciera Jicoténcal.32 Otro amigo de Heredia, José Teurbe Tolón, vivió en Nueva York en r824, mas de él no conocemos escrito alguno publicado en los Estados Unidos.

Como ya dijimos, Heredia tenía en su biblioteca un libro titulado Xicotencal, que aparentemente es la novela de Filadelfia. Sabemos también que al poeta cubano le atraía la figura del héroe tlaxcalteca. En febrero de 1823 , estando en Matanzas, comenzó a esbozar una tragedia sobre el tema, que lleva el nombre Xicoténcatl o los tlaxcaltecas. Dos de los cinco actos del drama, sin embargo, quedaron sin esbozar. ${ }^{33}$ Como es bien sabido, Heredia residió en Filadelfia en r824. Para 1826, sin embargo, ya se encontraba en México. El Ig de noviembre de ese año escribe a su amigo Del Monte: "Tal vez tienes razón en que escriba yo tragedias originales. Me he resuelto, aunque temeroso, y aún vacilo en la historia de la conquista entre Xicoténcatl y Cuatlpopoca. La última creo que vendrá por fin a ser la preferida".34 A pesar del interés que Heredia demostró

29 Estos libros vienen anunciados en la última página del T. II de jicoténcal. La novela El Vicario de W Whefield del Dr. Goldsmith aparece en la lista de libros que pertenecieron a Heredia.

30 Obra anunciada, como las anteriores, en la novela de Filadelfia.

31 Ver I. Centro M., La novela bispano-americana, Santiago, Chile, 1934, pp 9-10.

32 Ver Juan José Arrom, Estudios de literatura bispanoamericana, La Habana, 1950, pp. 93-70; también la Antología del Centenario, México, 1910, II, 1009

33 Ver Alonso E. Páez, Recordando a Heredia, La Habana, 1939, p. 55.

34 Carta reproducida por García Garófalo Mesa, Vida de José Maria Heredia en México, México, 1945, p. 247. 
por el tema, dudamos, por las siguientes razones, que él sea el autor de Jicoténcal. Al hablar de las poesías de Joaquín María de Castillo y Lanzas, poeta mexicano, Heredia hace esta crítica: "El lenguaje está muy lejos de ser puro. La fraseología cs en muchos trozos afrancesada". 35 En la novela que nos ocupa hay rasgos bien definidos del lenguaje afrancesado que critica Heredia y que no son característicos de su estilo. Al hacer Heredia la crítica de una poesía de otro poeta mexicano, Francisco Ortega, dice: "Queríamos que el señor Ortega hubiese economizado las acepciones del subjuntivo por pretérito imperfecto, que a pesar del ejemplo de Meléndez y otros modenos, no deja de ser arcaísmo que da al estilo un aire desagradable de afectación" (Ibid., p. I94). En la novela dicha construcción- "los tiempos remotos en que Tlascala fuera gobernada por un solo y poderoso cacique" ( $\mathrm{I}, \mathrm{II}$ ) - es usada con la mayor naturalidad. En general, el estilo cuidado y correcto de Heredia no tiene semejanza alguna con el af rancesado y a veces incorrecto del autor de jicoténcal. Además, Heredia conocía bien la obra de Walter Scott y, según parece, en la novela de Filadelfia no hay influencia del novelista escocés. ${ }^{36}$ Como sabemos, Heredia tradujo al español la novela $W$ averly, o Abora sesenta años de Scott. ${ }^{37}$

Manuel Lorenzo Vidaurre (I783-I84I), el inquieto peruano, publicó dos libros en Filadelfia en 1823 , sus Cartas americanas y su $P h_{\text {m }}$ del $P e$. rii, 38 ambos en la imprenta de J. F. Hurtel. Antes que pasara a Filadelfia había residido en Madrid y La Habana. Llegó a Nueva York el 13 de diciembre de 1822 e inmediatamente se trasladó a Filadelfia. Allí, nos dice en sus Carlas americanas (II, I87), "sólo el virtuoso Dn. José Antonio Isnaga previno mis necesidades". El estilo de Vidaurre no se parece al del autor de la novela de Filadelfia; en la ideología también hay grandes diferencias. Las Cartas americanas están dedicadas "Al serenísimo señor Don Francisco de Paula, infante de España", y en una dedicatoria que escribió para un libro del Dr. Villalobos, dirigida a Fernando VII, encontramos esta frase: "El nombre de V. M. formará su historia, y será lo mismo para la posteridad decir Fernando VII, que el príncipe justo, humano, sufrido, católico, el padre de los pueblos" (Cartas americanas, I, 52). Y

35 J. M. Chacón y Calvo (ed.), Revisiones literarias. La Habana, 1947, p. 125. (Reseña de Heredia publicada primero en su Miscelánea).

:36 Ver Anderson Imbert, "Notas..." p. 4. La opinión que Heredia tenía de Scott puede verse en su "Ensayo sobre la novela" en Revisiones literarias, citada, pp. $243-246$. Jal.).

37 México, 1833, 2 vols. (Ejemplar en la Biblioteca del Estado, Guadalajara,

3 A Ambas obras existẹn ẹn la Biblioteca del Congreso, Washington, D. C. 
también, al comentar la conducta de las tropas de Buenos Aires en Chile: "Siempre fui opuesto al sistema de la independencia, porque conocía sus resultas" (Ibid., p. 2II). En cuanto al estilo, Vidaurre usa constantemente la palabra cuasi en vez de casi, variante que jamás se halla en Jicoténcal. Además, en su obra hay errores crasos de ortografía, como sinco, usté, conbengo, precaber, etc.

Pasemos a hablar de un escritor cuyo nombre ha sido el único mencionado como posible autor de Jicoténcal. Trátase de don Vicente Rocafuerte, de quien los editores del Boletín Bïbliográfico Mexicano (enerofebrero, I951, p. vii), bajo el número 6970, dicen de la novela: "Es obra que merece un estudio. ¿Será su autor el inquieto Vicente Rocafuerte, que anduvo algún tiempo en Filadelfia y en donde publicó varios trabajos?". Nos parece que lo anterior es una mera conjetura, ya que, como hemos visto, eran varios los escritores que anduvieron algún tiempo en Filadelfia y que publicaron trabajos por allá. Sabemos que Rocafuerte estuvo en los Estados Unidos, y tal vez haya estado en Filadelfia - centro entonces de todos los conspiradores y refugiados políticos de España c Hispanoamérica-en I82I. Su Bosquejo ligerísimo no fue publicado, como ya vimos, en Filadelfia, sino en La Habana, en donde según parece se encontraba Rocafuerte en I822. Dos años más tarde pasa a Londres como representante del gobierno mexicano. En I 826 hace un viaje a México para asegurar la aprobación del tratad o de amistad entre México y la Gran Bretaña y vuelve a Londres en julio de 1827. En los Estados Unidos publicó tres libros, dos en Filadelfia y uno en Nueva York. Las Ideas necesarias a todo pueblo americano independiente, que quiera ser libre y la Memoria político-instructiva vieron la luz en Filadelfia en I82I, la primera en la imprenta de D. Huntington y la segunda en la de J. F. Hurtel. El Ensayo política, obra sobre el gobierno colombiano, apareció en Nueva York en 1823, en la imprenta de A. Paul. ${ }^{39}$ Según parece, la Memoric político-instructiva pertenece a Fray Servando Teresa de Mier y no a Rocafuerte. El P. Mier había vivido en Filadelfia de junio (o julio) de I82I a febrero de I822. Allí habia también publicado su Bosquejo ligerisimo de la revolución de Mégico en este año de 1822.40 Examinando las obras de Rocafuerte, no encontramos mucha semejanza entre su estilo

ton, D. C.
40 Ver J. M. Miquel i Verges, "Aspectos inéditos de la vida de fray Servando
en Filadelfia", Cuadernos Americanos, Año V, vol pp. 187-205. 
y el de la novela. El único rasgo estilístico usado por ambos autores es la frase en el interim (en vez de mientras tanto), que se repite con insistencia en Jicoléncal y que hemos encontrado en algunas cartas de Rocafuerte. ${ }^{40}$ En cambio, escribe la palabra megicano con $g$ y no con $j$. Nos parece arriesgado adjudicar la paternidad literaria de la obra a base de tan sutiles semejanzas. Por lo tanto, debemos eliminar su nombre de la lista de posibles autores, a no ser que un estudio más detallado revele nuevas semejanzas, o se descubran nuevos datos.

Fáltanos estudiar el caso de José Antonio $\mathrm{Saco}_{2}$ escritor cubano que, en I824, abandona su patria y se refugia en Filadelfia, en donde publica, en 1826 y precisamente en la imprenta de Stavely, su traducción de los Ele. mentos de dierecho romano de J. Heineccio. ${ }^{42}$ En diciembre del mismo año Saco pasa a Nueva York y, tras corta visita a Cuba, vuelve a los Estados Unidos. Con el P. Varela funda en Nueva York el Mensajero semanal ( 3 tomos, I828-I83I), en el cual colaboran otros cubanos, entre ellos Gaspar Betancourt Cisneros, "El Lugareño".43 Al leer las obras de Saco y comparar su estilo con el del autor de ficoténcal nos damos cuenta de que nada tienen en común. No nos parece, por lo tanto, posible que sea él el autor de la novela. ${ }^{4 / 4}$

Pasemos, en fin, a estudiar el caso de otro escritor cubano que, si no podemos decir con seguridad absoluta que es el autor de ficoténcal, la evidencia que hemos recogido apunta en esa dirección. Trátase del P. Félix Varela (1788-1853), quien vivió en Filadelfia en 1824. Su biógrafo, José Ignacio Rodríguez, ${ }^{\mid 45}$ nos dice:

41 Ver El empréstito de México a Colombia, Introd. y Notas de Joaquín Ramírez Cabañas, México, 1930 (Archivo bistórico diplomático mexicano, No. 33), pp. 40 v 237.

42 Véase la "Introducción" a su Colección de papeles cientificos, bistóricos, politicos y de otros ramos sobre la Isla de Cuba, París, 1858, I, también las pp. 282 y 316.

43 Ver Calcagno, p. 109. Su biógrafo, Federico Córdova (Gaspar Betancourt Cisneros, El Lugareño, La Habana, 1938), duda que haya colaborado en el Mensajero (vide p. 88). El Lugareño residió en los Estados Unidos de 1822 a 1834; sin embargo, antes de 1826 no había publicado nada; es escritor de formación tardía y dudamos que sea el autor de Jicoténcal; su estilo no es el del autor de la novela.

44 Véanse, además de su Colección de papeles... (buena obra para estudiar su estilo, ya que allí se recogen obras de varias épocas), su Historia de la esclavitud de la raza africana..., Habana, 1883 (nueva ed., con prólogo de Fernando Ortiz, Habana, 1838, 4 tomos); también ver José Antonio Saco, documentos para su vida, anotados por Domingo Figarola-Caneda, Habana, 1921.

45 Vida dẹl Presbitẹro don Fẹlix Varela, Nueva York, 1878, 
Poco después de haber llegado a Nueva York se dirigió el P. Varela a Filadelfia... Allí vivió en los primeros meses de 1824, en la casa número 224 de Spruce Street, ocupada por la señora que se llamaba Mrs. Frazier; y allí publicó la primera producción suya que vió la luz en los Estados Unidos. Pero pronto cambió de pensamiento y regresó a Nueva York... Durante su permanencia en Filadelfia publicó allí, imprimiéndolos en el establecimiento de los señores Stanley [sic] y Bringhurst, número 70 de la calle 3a. del Sud, los tres primeros números de un periódico en castellano, que se denominaba El Habanero, papel político... (p. 229).

La imprenta donde se publicó El Habanero es, por supuesto, la misma en que se publicó Jicoténcal, esto es, la de Stavely, como podemos ver en la portada de la revista. Dos de los tres primeros números de El Habanero aparecieron en I824, y el tercero en I825. El número 4, también en I825, se publica ya en Nueva York. En la misma imprenta de Stavely y Bringhurst publicó el P. Varela otra obra, la 2a. edición, corregida y aumentada, de sus Lecciones de filosofía, en tres volúmenes, año de $1824 .{ }^{46}$

¿Por qué publicó Varela sus obras en la imprenta de Stavely? Según parece, este impresor era episcopalista y Varela, como católico, veía con simpatía a los miembros de esa secta religiosa. En sus Cartas a Elpidio leemos: "En cuanto a los episcopales o sectarios de la iglesia de Inglaterra, casi son católicos". ${ }^{47}$ Existe, además, otra coincidencia que tal vez sea significativa. El P. Varela vivía, en Filadelfia, en el número 224 de la calle Spruce. El Dr. Frederick Huttner, que fue quien registró la novela, como ya hemos visto, en el número 239 de la misma calle. ¿Trabó el P. Varela amistad con este médico y le dejó el manuscrito de la novela? Es esta una pregunta que casi es imposible contestar. Sea como fuere, el caso es que, entre todos los escritores que hemos estudiado, el estilo y la ideología de Varela son los que más se parecen a los del autor de ficoténcal. Comparemos primero los estilos.

En la ortografía existen algunas semejanzas; la palabra acia se encuentra escrita $\sin b$ en la novela. En una interesante reseña que el P. Varela publicó sobre las Poesías (Nueva York, I825) de Heredia, en el periódico New York American en 1825,48 encontramos la nalabra acia también es-

146 Ejemplar en la Biblioteca del Congreso, Washington, D. C. Heredia publicó en México, en El lyis (II, 1826, p. 28), un anuncio literario sobre esta $2 a$. ed. (Ver Revisiones literanas, p. 118). 112 .

47 Félix Varela y Morales, Cantas a Elpidio, 2 tomos, La Habana, 1944, II,

48 Vol. VI, Núm. 1657, viernes 6 de agosto de 1825, p. 2, col. 1, 2. Reproducida en More, "José María Heredia en Nueva York", citada, pp. 288-291. 
crita $\sin h$. Pero es en el léxico, sin embargo, donde abundan las semejanzas; como ejemplos hemos seleccionado el uso de las siguientes palabras, y no son las únicas.

\section{Jicoténcal}

si me apartara un ápice (I, 194)

en las aras del amor (II, 226)

Teutila fue... a recibit los baldones

(II, 11)

cobonestar su violencia (II, 9)

con una contra-revolución apagó el primer tumulto $(\mathrm{I}, 88)$

el Cordero inmaculado (I, 36)

por cuimulo de males (II, 18)

les parece que se va a desquiciar $\mathrm{dl}$ mundo (II, 60)

ideas... diametralmente opuestas (I, 163)

se empeñó una batalla tan sangrienta... (I, 95)

la prostitución y envilecimiento

lo toman como garante de su seguridad (II, 32)

derechos bollados por un tirano (II, 15)

la imperiosa lei de la fuerza $(\mathrm{I}, 85)$

crímenes inauditos (II, 240)

vil e inicuo (II, 91)

providencia liberticida $(I, 122)$

¡La perfidic unida a la franqueza! (I, 188)

he proyectado... (I, 125)

los satélites que lo rodean (II, 207)

un hombre $\tan$ virtuoso $\mathrm{y} \tan$ sensible (II, 91)

la tea de la discordia (II, 36)

encendiendo los odios (I, 57)

la llama del amor patrio (I, 168)

ideas ridículas $(\mathrm{I}, 27)$
Varela 49

ni un apice $(H, 161)$

en aras del poder (E, II, 43)

y llenan de baldones al ilustre pattiota $(\mathrm{H}, 137)$

cobonestar sus desvaríos (E, I, 6)

las más enérgicas contrarrevoluciones

$(\mathrm{H}, 139)$

el Cordero de Dios $(\mathrm{R}, 85)$

un cúmulo de pensamientos $(\mathrm{M}, 242)$

Desquiciado el edificio... (E, II, 90)

sentido diametralmente contrario $(\mathrm{H}$,

197)

el choque de la libertad contra el despotismo va a empeñarse de un modo terrible ( $E, 137)$

hasta el envilecimiento $(\mathrm{H}, 207)$

sin salir garante de este hecho (E, II, 103)

la impiedad. . . todo lo bolla (E, I, 89)

la imperiosa ley de la necesidad $(\mathrm{H}$, 64)

portentos inauditos (E, II, 87)

inicuas opresiones $(\mathrm{R}, 409)$

crueles liberticidas $(\mathrm{H}, 129)$

la más negra perfidia $(\mathrm{H}, 3)$

proyectáronse empresas $(\mathrm{H}, 207)$

de quien sería un mero satélite $(\mathrm{E}, \mathrm{II}$, 102)

toda alma sensible $(H, 62)$

la tea fúnebre $(\mathrm{R}, 98)$

sólo sirven para encender más el odio

$(\mathrm{H}, 31)$

el fuego del amor patrio $(\mathrm{H}, 85)$

epitetos ridículos $(\mathrm{H}, 172)$

49 Al citar a Varela hemos usado las siguientes siglas: $\mathrm{H}-$ El Habanero, La Habana, 1945; E-Cartas a Elpidio, ed citada; M-Miscelánea filosófica, La Habana, 1945; R-Rodriguez, Vida del Presbitero don Félix Varela, ed, citada. 
Otras semejanzas en el estilo las hallamos en el uso del artículo con nombres geográficos que por lo general no lo llevan:

una hermosa parte de la América (I, a nadie se oculta todo lo que puede ser 1) la América $(\mathrm{H}, 82)$

las largas guerras que la España sostuvo contra los moros (I, 81)

entre tanto, la España ocupada por un ejército extranjero $(\mathrm{H}, 56)$

Además, el uso del pronombre la como dativo femenino:

El buen anciano hizo traición a su patria, creyendo que la rendía un gran servicio $(\mathrm{I}, 90)$

$Y$ manda pedir a ésta el permiso de hablarla (I, 192)
A no ser que usted de a esta palabra la acepción que $l a$ dan los déspotas $(\mathrm{H}$, 117)

Lejos de sobrarla, fáltanla brazos $(\mathrm{H}$, 180)

Tanto Varela como el autor de Jicoténcal usan del término filósofo para referirse a sí mismos; en las siguientes citas hay similitud también en la exposición de las ideas:

Todas las naciones han tenido épocas de gloria $y$ de envilecimiento; y algunas veces han pasado de uno a otro de estos extremos con tanta rápidez, que al volver una página de su historia le parece al lector que se le habla de otro siglo y de otro pueblo.

El filósofo que examina con imparcialidad estos grandes sucesos, encuentra su causa en el influjo que ejercen sobre los pueblos las virtudes o los vicios $(I, 155)$.

El ojo perspicaz del filósofo sabe distinguir, entre el fango y la basura que ensucian el papel de las historias, algunas chispas de verdad (II, 169)

La historia es sin duda la maestra de la vida, y un depósito inagotable de objetos dignos de la contemplación de un filósofo $(\mathrm{M}, 78)$

En términos generales, podríamos decir que la prosa de la novela se parece a la de Varela en el estilo discursivo, aforístico, de giro rápido y conciso. Ambos autores tienen una inalterable noción de lo justo $y$, con frecuencia, expresan sus ideas de la misma manera. Mas es en la ideología, sin embargo, donde encontramos mayor semejanza. En Jicoténcal no hay ninguna idea - moral, religiosa o filosófica-que no pudiera ser atribuida al P. Varela. Al mismo tiempo, en las obras del filósofo cubano no hemos hallado ideas que contradigan las de la novela. Discutiremos las que nos parecen de mayor importancia.

La actitud ante el indigena americano es la misma; para el autor de ficoténcal los indígenas "sụcumbierọn a las artes e intrigas europeas, que 
un puñado de ambiciosos supo manejar contra su sencillez y contra su diferente manera de vivir" (I, 6-7). Teutila, que simboliza la inocencia, es "un alma sencilla, no corrompida por las artes de la civilización" (I, 46). En el ya mencionado articulo sobre las poesías de Heredia, Varela nos dice que ese autor "deja correr su fértil imaginación por las escenas americanas, y ya presente a nuestra vista los interesantes cuadros que la naturaleza ofrece en el nuevo hemisferio, ya declame contra los crueles opresores, ya elogie el amable carảcter y las virtudes de sus naturales, su estilo siempre enérgico, fácil y variado".

La novela de Filadelfia es una alabanza a la libertad - simbolizada por los dos Jicoténcal-y, a la vez, una acerva censura de los déspotas y los tiranos, simbolizados por Cortés y Moctezuma. El humanismo del atttor se manifiesta en este discurso:

Cuando se encarecen como heroicas y grandes hazañas la devastación de pueblos enteros; la agresión injusta de países pacíficos y remotos; la muette y la desolación conducidas por un ambicioso, y acompañadas de todos los crimenes y horrores de una soldadesca sin freno; cuando se veneran como hechos de la piedad más cristiana el haber levantado una cruz sobre los escombros de provincias enteras y sobre los cadáveres de millones de hombres; y el haber convertido a algunos naturales, arrastrados o por el miedo, o por la bajeza, o por el interés... ¡ se osa profanar asî el nombre augusto de Libertad! (II, 193).

Varela, en su reseña del libro de Heredia, nos dice que este poeta "presenta la amable libertad y el tremendo monstruo de la tiranía con los colores más vivos"; en sus Cartas a Elpidio nos habla de los "infieles a la noble causa de la justicia y santa libertad" (I, 54), y alli mismo caracteriza a los déspotas como "infieles a la noble causa de la justicia y santa libertad" (I, 53). El autor de la novela, hablando de los tiranos, dice: " $\mathrm{S}$ Sin duda un Ser poderosísimo conserva la especie humana, que la vemos sobrevivir a semejantes monstruos!" (II, 196). De Maxiscatzin, hombre traidor e inmoral, el viejo Jicoténcal dice: “ Desgraciados de nosotros si un perverso quiere abusar de la credulidad del vulgo inocente!" (I, 69). Varela, hablando de los falsos patriotas, observa: "Es desgraciada toda sociedad, grande o pequeña, donde tienen influjo y aprecio hombres inmorales. Muchos aspiran a este título de patriotas entre la gente incauta e ignorante" $(H, 6)$. En la novela se condena a los tiranos y a los déspotas no por ser españoles - dos de ellos, Maxiscatzin y Moctezuma, son indigenas- sino por sus acciones. El mexicano Teutile le dice a Jicotén- 
cal, refiriéndose a Cortés: "Si ese estrangero cuyas prendas me admiran, se hubiera puesto de parte de la justicia y de la equidad; si nos hubiera dado ejemplos de moderación, de sabiduría y de virtud; ¿qué importa de la parte que nos viniera el bien, como los pueblos fueran felices?" (II, 20). Varela, en su ensayo "Amor de los americanos a la independencia" publicado en El Habanero, expresa la misma idea: "Por un error funesto o por una malicia execrable suele suponerse que el amor a la independencia en los americanos proviene de su odio a los curopeos, y no que este odio se exita por el mismo amor a la independencia y por los esfuerzos que suelen hacer los europeos para que no se consiga. Los americanos tienen por enemigos a los anti independientes, sean de la parte del mundo que fueren, y aprecian a todos los que propenden a su libertad aunque fuesen hijos del mismo Hernán Cortés... Porque el odio no es a las personas sino a la causa que sostienen" ( $\mathrm{H}, 8 \mathrm{I})$.

En las ideas filosóficas y religiosas del autor de ficoténcal es aparente la influencia del racionalismo de la Ilustración francesa. De las supersticiones, el viejo Jicoténcal dice: "Un milagro es una cosa imposible, y el creerlo ofende la sabiduría y el poder de ese mismo Dios que tú Fr. Bartolomé de Olmedo llamas infinitamente sabio y poderoso. Todo lo que nuestra inteligencia alcanza a conocer en este mundo, está ordenado por leyes inmutables, y con una relación tan íntima que cualquiera de éstas que se infrinjan, faltaría enteramente el orden de las cosas" (II, 53). El P. Varela sostenía ideas semejantes: en su Miscelánea leemos: "Un plan sistemático es un plan absurdo. La naturaleza no conoce estas normas. Inventar un sistema y buscarle pruebas, es un delirio; observar efectos y deducir causas, ésta es una ciencia" (p. I73). A la vez, nos habla de la "imperiosa voz de la naturaleza" $(H, 86)$, de la "imperiosa ley de la necesidad" $(H, 64)$, de la "imperiosa voz de la razón $(H, 62)$ y, hablando de las supersticiones: "A sangre fría sacrifica este monstruo innumerables víctimas para honrar a Dios, cuya clemencia en nada se demuestra tanto como en no arrojar rayos que destruyan a estos crueles profanadores de su santo nombre" ( $\mathrm{E}, \mathrm{II}, 90) .50$

De la justicia, base de la moral, leemos en Jicoténcal. "La justicia es

50 Sobre las ideas filosóficas de Varela véanse los libros Varela y la reforma filosófica en Cuba (La Habana, 1942) de A. Hernández Travieso, y El pensamiento de Félix Varela y la formación de la conciencia cubana (La Habana, 1950) de Rosario Rexach. Este último autor considera a Varela como escritor de transición, ya que, aunque acepta la filosofía de los empiristas ingleses y los ideólogos franceses, no abandona el dogma de la iglesia catốlica. Esta es precisamente la actitud del autor de Jicoténcal. 
la única regla que debe rejir todos los intereses de todas las causas; y sin ella no hay ni política ni gobierno, sino despotismo, desorden y tiranía" (I, 2II). Según Varela, "la justicia nos prescribe dar a cada uno lo que le corresponde, y es la virtud que sostiene la sociedad" ( $R, 68)$. Y tam. bién: "El deber, pues, del verdadero sacerdote, del verdadero ministro del Dios justo, es exaltar, defender, sostener la justicia... y trabajar en retirar al hombre del yugo arbitrario del hombre para ponerlo bajo la protección de la justicia y de la fe divina" ( $R, 2$ I5).

Encontramos también en la novela, además de las semejanzas en el estilo y la ideología, un trozo que tal vez revele un episodio acontecido al P. Varela en Filadelfia. Cuando los primeros números de El Habanero llegaron a Cuba, su lectura causó tanto disgusto en los círculos del gobierno que enviaron a un asesino pagado expresamente para matar al P. Varela. Tal vez a ello se deba que no se haya quedado el filósofo cubano en esa ciudad, sino que pasó a Nueva York. En el suplemento al número 3 de El Habanero ( 1825 ) el P. Varela dice: "Acabo de recibir noticia de que en consecuencia de los efectos producidos por el segundo número, se ha hecho suscripción para pagar asesinos que ya han encontrado y que deben venir de la isla de Cuba a este país sin otro objeto que este asesinato... ¡ $\mathrm{Mi}$ serables! ¿Creéis destruir la verdad asesinando al que la dice?" (H, I52). El libro VI, o sea el último, de la novela abre de esta manera: "Cuando el poder arbitratio llega a asesinar a un hombre virtuoso, cubriendo este horrible atentado con una farsa judicial, tan ridícula como insultante; $y$ cuando el despotismo descarga así su mano de hierro a presencia de un pueblo que no le ahoga o despedaza en la justa indignación que debe escitar tan bárbara tiranía; ese pueblo sufre justamente sus cadenas; y aun éstas son poco para lo que merece su cobarde y vil paciencia" (II, I67).

Fáltanos, por fin, examinar algunas observaciones que el profesor Anderson Imbert hizo con referencia al autor. En la nota 5 de su artículo, ya citado, nos dice: "Valdría la pena que se investigara a fondo este pequeño misterio del autor anónimo de Iicoténcal. Mis intentos, emprendidos en Filadelfia y en México, no dieron resultado. Me inclino a sospechar que fue español: uno de tantos indicios estilísticos es que habla de 'nuestros antepasados' al referirse a los españoles y ¿con perspectiva europea? califica a México de 'país remoto'". Tratándose del P. Varela, en caso de 
que tengamos razón y él sea el autor de la novela, no nos parece extraño que llame a los conquistadores "nuestros antepasados". Hay que tener presente que en I826 Cuba pertenecía a España y que los cubanos eran ciudadanos españoles. La misma cita podría ser utilizada para afirmar que el autor es mexicano, ya que se podría decir que los conquistadores son los antepasados de los mexicanos. En cuanto a la referencia que se hace de México como "país remoto", el profesor Anderson Imbert no menciona la página donde se encuentra. Tal vez se trate de esta frase, la única que hemos encontrado en la novela: "Cuando se encarecen como heroicas y grandes hazañas la devastación de pueblos enteros; la agresión injusta de países pacíficos y remotos..." (II, I93). Aquí, el autor está pensando acerca de la conquista de México por España; y se podría agregar, la conquista del Perú también. España, en verdad, conquistó pueblos remotos. El autor, es evidente, no piensa en México como país remoto para él, sino para el gobierno que lo conquistó.

Podría objetarse que las semejanzas ideológicas entre el autor de la novela y el $P$. Varela que hemos anotado podrían existir también entre la novela y las obras de otro autor, ya que dichas ideas eran comunes entre los pensadores liberales de la época; mas es también verdad que existían diferencias radicales, sobre todo cuando se trataba de las relaciones entre España y la América Española. Lo que podemos decir es que, de todos los escritores que hemos examinado, las ideas de Varela son las que van más de acuerdo con las de la novela. Las mismas observaciones podrían ha. cerse en cuanto al estilo.

Una objeción más. Podría decirse que no se conocen obras de ficción del P. Varela. Esto es verdad. Sin embargo, en la obra de Rexach leemos: "Y también distraía sus ocios con el arte. Alguna vez parece haber escrito una pequeña obra teatral" (p. 68). Y si escribió una obra teatral, ¿por qué no una novela? Que no la haya publicado bajo su nombre es fácil de comprender, ya que el P. Varela era por aquellos años el maestro de las juventudes hispanoamericanas, además de ser padre de la Iglesia. Sus discípulos — Saco, Luz y Caballero, Heredia, Delmonte, Miralla- no se lo hubieran perdonado.

Tal vez en el futuro se descubran nuevos datos acerca de la primera novela histórica en castellano. Nosotros mismos pensamos seguir haciendo 
investigaciones en torno al problema. Mientras tanto, y por las razones arriba expuestas, proponemos el nombre del P. Félix Varela como el del posible autor de Jicoténcal.

LUIS LEAL

University of Illinois

Urbana Illinois 
